\title{
A Rule-Based and MT-Oriented Approach to Prepositional Phrase Attachment
}

\author{
Kuang-hua Chen and Hsin-Hsi Chen \\ Department of Computer Science and Information Engineering \\ National 'laiwan University \\ 1, SHC. 4, Roosevelt RI)., 'Taipei \\ 'AIWAN, R.O.C. \\ khchen@unlg.csie.ntu.edu.tw, hh_chen(i)csic.ntu.edu.tw
}

\begin{abstract}
Prepositional Phrase is the koy issue in structural ambiguity. Recently, researches in corpora provide the lexical cue of prepositions with other words and the information could be used to partly resolve ambiguity resulted from prepositional phrascs. 'I'wo possible attachments are considered in the literature: either noun atiactument or verb attachment. In this paper, we consider the problem from viewpoint of machine translation. Four different attarhments are told out according to their functionality: noun attachnent, verb attachment, sentence-level attachment, and predicate-level at,tachment. Both lexical knowledge and semantic knowledge are involved resolving attachment in the proposed mechanism. Hxperimental results show that considering more types of prepositional phrases is useful in machine translation.
\end{abstract}

\section{Introduction}

Prepositional phrases are usually ambiguous. 'I'he well-known sentence shown in the following is a good example.

Kevin watched the girl with a telescope

Whether the prepositional phrase with a telescope modifies the head noun girl or the verb watch are not resolved by using only one knowledge source. Many researchers olserve text corpora and learn some knowledge based on language model to determine the plausible attachment. For example, we could expect that the aforementioned prepositional phrase is usually attached to verb according to text corpora. However, the correct attachment is dependent on world knowledge sometimes.
Some approaches to determination of Pl's are reported in literature (Kimball, 1973; Frazier, 1978; Ford et al., 1982; Shieber, 1983; Wilks of al., 1985; Liu et al., 1990; (then and Chon, 1992; Iindle and Rooth, 1993; Brill and Resnik, 1994). The possible attachment they consider are NOUN attachment and VERIB attachment. These resolutions fall into three categories: syntax-based, semantics-based and corpus-based approaches. The brief discussion are described in the following:

1. Syntax-based

- Right Association (Kimball, 1973)

The PPs always modifies the nearest. component proceding it.

- Minimal Atachmont (Frarier, 1978: Shicber: 1.988)

'The correct attaching point of a PP' in a sentence is determined by the number of nodes in a parsing tree.

2. Semantics-based

- Icxical Preference (Ford et al., 1982) 'The real attaching point must satisfy some constraints, c.g., verb foatures. Different verlos accompanying with the same PPs may have the different attaching points.

- Preference Semantics (Wilks et al., 1985) Wilks and his colleagues argue the real attaching point must be determined by the preference of verbs and prepositions.

- Propagated Semantics (Chen and Chen, $1092)$

The attachment of prepositional phrase is co-determined by the semantic usage of noun, verb, and preposition.

3. Corpus-based

- Statistical Score (Liu et al., 1990)

They use semantic score and syntactic 
scope to determine the attakhing point. 'llese seores are trained from text cor poria.

- Lexical Astoriation (Mindle and Rooth. 1999:3)

'This method applies statistionl techniques to discover the Inxical assoctation from text colpora. 'Thus, the at tachmont of PPs is determined.

- Model Refincment (Bzill and Rosulk, 1094)

'Their approach assumes every P' lnod ifies the inumediatedy previous noun and uses rules trained from lext coppora to diange the cromeous altialuments.

f'hese approaches manage to besolve the Pl' at. tachment via only one language comsidemalion. In contrast, wo investigate this problem from view. point of marhine tremstation and do mot resteriet onrselves in two possible at anelument choiers

In the sections what follows, we will first present. our viewpoint from machine translation to this probletu. Section 3 will cliscussis the detail resofution to P'Ps attachnent, which rensideles more different attandmonts. Section 4 will conduct experiments to investigate ons approath. Sordion t) will provide some concluding remarks

\section{Our Viewpoint from M'T}

from the viewpoint of machine transtation, in particular, linglish-Chinese mathine transiation (chen and ('ben, 1995), the main shorterenting of the approaches mentioned in previons section is that they all consider cithes P'Ps modify nom or P's modify verbs. Although P's usually modify nomes or verls, there are some comber examples even in the simple sentences like "there is a book on the talule" and "the apple has wom in it". In the first example, the Pl" "on the tablele" is nei. ther used to modify the copula verb nor the noun phrase "a book". It deseribes the situation of the whole sentence. The second example shows that the Pl" "in it" is also not a modifier, but a complement to the preceding noun phrase. 'That is, the PI lias a nonrestrictive usage. To transfer PPs among differont languages, we must apture the correct interpretation. 'Therefore, wo distinguish four different prepositional phrases.

- Predicative P'Ps (PPP): P'Ps that serve as predicates.

$\mathrm{He}$ is at home

Tal zai4 jia1.

He found a lion in the net.

Tal falxian4 shilzi5 zai4 wang $3 z i 5$ li3.
- Sontential PPs (SPP): PP's that serve functions of tine and localion.

There is no parking along the street

The4 tiao2 jiel shang4 jintzhi3 ting2 che 1

We had a good time in Paris.

Zai4 balli2 wo 3 men 5 you 3 yil duan 4 mei3hao 3 de5 shi2guang1.

- Pl's Modifying Vorlos (VPP)

I went to a movie with Mary.

Wo3 han4 ma3lis qu4 kan4 dian4ying3

I bought a book for Mary.

Wo3 wei4 ma3lit mai3 les yi4 ben 3 shul

- Pl's Modifying Nouns (NIP)

The man with a hat is my brother.

Dai4 mao4zi5 de5 ren'? shi4 wo3 gelge5

Give me the book on the desk.

Ba3 zhuo1 shang4 deb shu 1 gei3 wo 3 .

It. is ofverous that these four diflepent proposi tional phrases have their own appropriale positions in chinesse. 'llat is alter we determine the type of a prepositional phase, the constituent to

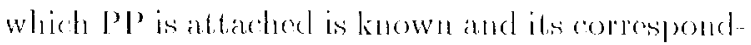
ing position in chineso is also determined.

\section{Resolving PP-Attachment}

In the previons section, four different types of P's are defined areording to their functionality. 'llus, the resolution to this problen is to deter mine which type the Pl's belong too. 'The basie stepsis are:

- ('heck if it is a Pl'P.

- cherek if it is an silp

- Check if it is a VPP.

- Otherwise, it is an NPl'

Now, the problem is whate comstitutes the mech anism of cach stop.

Oxford Advaneed Leatmer's Dictionatry (OAID) (llornby, 1989) defines 32 different vorb patterms to describe the usige of verles. These verb frames are like sketeton of a sentenee and the constituents are the flesh of sentence, (hen and Chen (1994) have proposed a method to determinate the predicate argument structure of a sentence. The OALD-defincel verb frames are regareded as the primary language knowledgo souree and an NP parser and a linte-stalo mochanism are cooperatively used to determine the phatl. sible predicate-argument structure. Onee the predicate-argument structure of a sentence contains prepostional phrase, the maderlyiug prepositional phrase is P'P'. 
As for SPP, VPP, and NPP, the rules arc dependent on the lexical knowledge and semantic usage. That is to say, the semantic tag should be assigned to each word. Figure 1 and Figure 2 describe the semantic hierarchy for noun and verb. However, manually building a lexicon with semantic tag information is a time-consuming and humanintensive work. Fortunately, an on-line thesaurus provides this information. Roget's thesaurus defines a semantic hierarchy with 1000 leaf nodes shown in Table 1. liach leaf node contain words with this semantic usage, that is, thesc words have the semantic tags represented by these leaf nodes. We just map those leaf nodes to the semantic definitions listed in Figure 1 and Figure 2. Therefore, nouns and verbs in ruming texts could be easily assigned scmantic tags in our semantic definitions.

In general, four factors contribute the determination of PP-attachment: 1) verbs; 2) accusative nouns; 3) prepositions; and 4) oblique nouns. We use a 1-tuple $\langle V, N 1, P, N 2\rangle$ to denote the relationship of a possible $P P$ attachment, where $\mathrm{V}$ denotes semantic tag of verbs, N1 denotes the semantic tag of accusative noun, $\mathrm{P}$ denotes the preposition and $\mathrm{N} 2$ denotes the semantic tag of oblique noun. For example, the following sentence has the 4-tuple $\langle$ non_speech_act, human, with, instrument $\rangle$.

Kevin watched the girl with a telescope.

Having the 4-tuple in advance, we could apply 67 rule-templates listed in $\Lambda$ ppendix to determine what the PP type is by aforementioned steps. That is, apply SPP rule-template first, and then VPP rule-template. If none succods, the PP should be an NPP. We summarize the algorithm as follows.

\section{Algorithm 1:}

\section{Resolution to PP-Attachment}

(1) Check if it is a PPP according to the predicate-argument structure.

(2) Check if it is an SPP according to 21 ruletemplates for SPP.

(3) Check if it is a VPP according to 46 ruletemplates for VPP.

(4) Otherwise, it is an NPl'

\section{Experiments}

The Penn Treebank (Marcus et al., 1993) is used as the testing corpus. The following is a real example extracted from this treebank.
(

(S (ADVP (NP Next week))

(S

(NP (NP some inmates)

(VP released

(ADVP early)

(PP from

(NP the Hampton County jail.

(PP in

(NP Springfield))))))

will be

(VP wearing

(NP (NP a wristband)

(SBARQ

(WHNP that)

(S (NP T)

(VP hooks up

(pp with

(NP a special jack

(PP on

(NP their home phones)

)) )) )) )

The PPs contained in Penn 'Treebank are collected and associated with one label of PPP, SPP, VPI, or NPP. For example, the PI's contained in the aforementioned sentence are extracted as follows.

〈from the Hampton County jail, VPP〉

$\langle$ in Springfield, NPP〉

$\langle$ with a special jack, VPP

$\langle$ on their home phones, NPP

These extracted PPs constitute the standard set and then the attachment algorithm shown in previous section are applied to attaching these PPs. Finally, the attached PPs are compared to the standard set for performance cvaluation. 'The results are shown in Table 2.

\begin{tabular}{|l|c|c|}
\hline & Total & Correct \\
\hline SPP & 750 & 750 \\
\hline VPP & 6392 & 4923 \\
\hline NPP & 7230 & 7230 \\
\hline PPP & 387 & 387 \\
\hline Total & 14759 & 13290 \\
\hline
\end{tabular}

'Table 2: Expcrimental Results

First, NPP and VPP dominale the distribution of PPs (92\%). 'The former occupies $49 \%$ popula tion and the latter $43 \%$. If we carelully process NPP and VPP, the result, would be good. In lact, the proposed algorithm is based on the philosophy of model refinement. That is, we assume ach PP is NPP except it is a PPP or it matches the 67 rule-templates. Table 2 shows that cach NPP is 


\begin{tabular}{|c|c|c|c|c|c|}
\hline CI $\triangle S S$ & SlCOTON & $\operatorname{TAC}$ & CI. $\Lambda \mathrm{SS}$ & STOYION & $\bar{\top} \Lambda($ \\
\hline \multirow{8}{*}{$\begin{array}{l}\text { ABSSIRAO'I } \\
\text { RIILA'IIONS }\end{array}$} & lexistence & 1.8 & \multirow{4}{*}{$S P \Lambda C H$} & In General & 180.191 \\
\hline & Relation & $9 \cdot 24$ & & Dimensions & 192239 \\
\hline & Quantity & $25 \quad 57$ & & lorm & $240-263$ \\
\hline & Order & 5883 & & Motion & 264315 \\
\hline & Number & 81105 & \multirow{3}{*}{ MAITLR } & In General & $316 \quad 320$ \\
\hline & Tine & $106-139$ & & Inorganic: & 321356 \\
\hline & Chajgo & 140.152 & & Organic: & $357.4 \overline{49}$ \\
\hline & Gatusation & 158179 & \multirow{5}{*}{ AFUECIIONS } & Tu Gencral & $820 \overline{826}$ \\
\hline \multirow[t]{2}{*}{ TNTELTLCT } & Tommation of Ideas & $150-515$ & & Personal & $8 \overline{27} 8 \overline{87}$ \\
\hline & Communication of ldoas & $516 \cdot 509$ & & Sympathetic: & 888.921 \\
\hline \multirow[t]{2}{*}{ VOLITON } & Individual & $6(30) 736$ & & Moral & $922 \overline{975}$ \\
\hline & Intersocial & 737819 & & Religious & 9751000 \\
\hline
\end{tabular}

'Table 1: Classification of Roget's 'Thesaurus

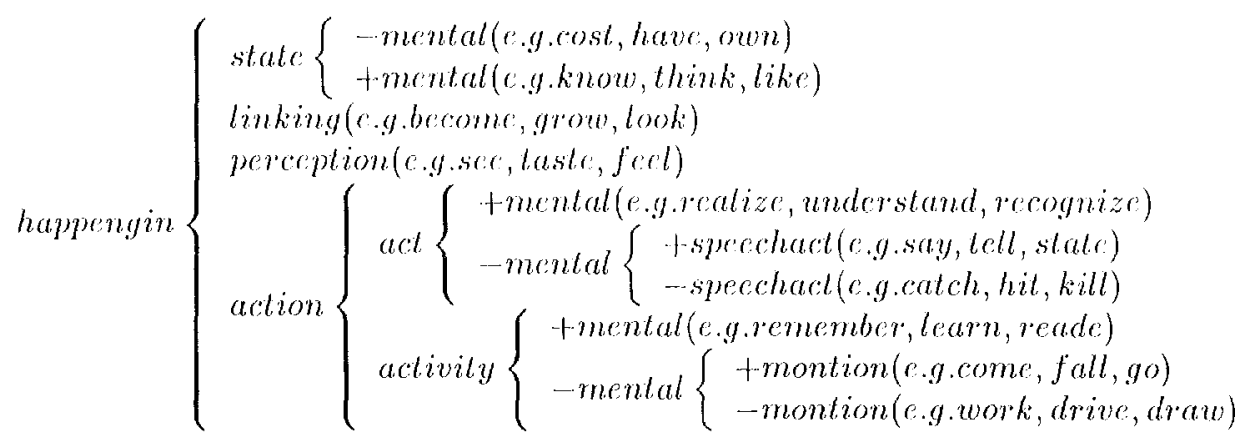

Figure 1: Semantics lags for Verbs.

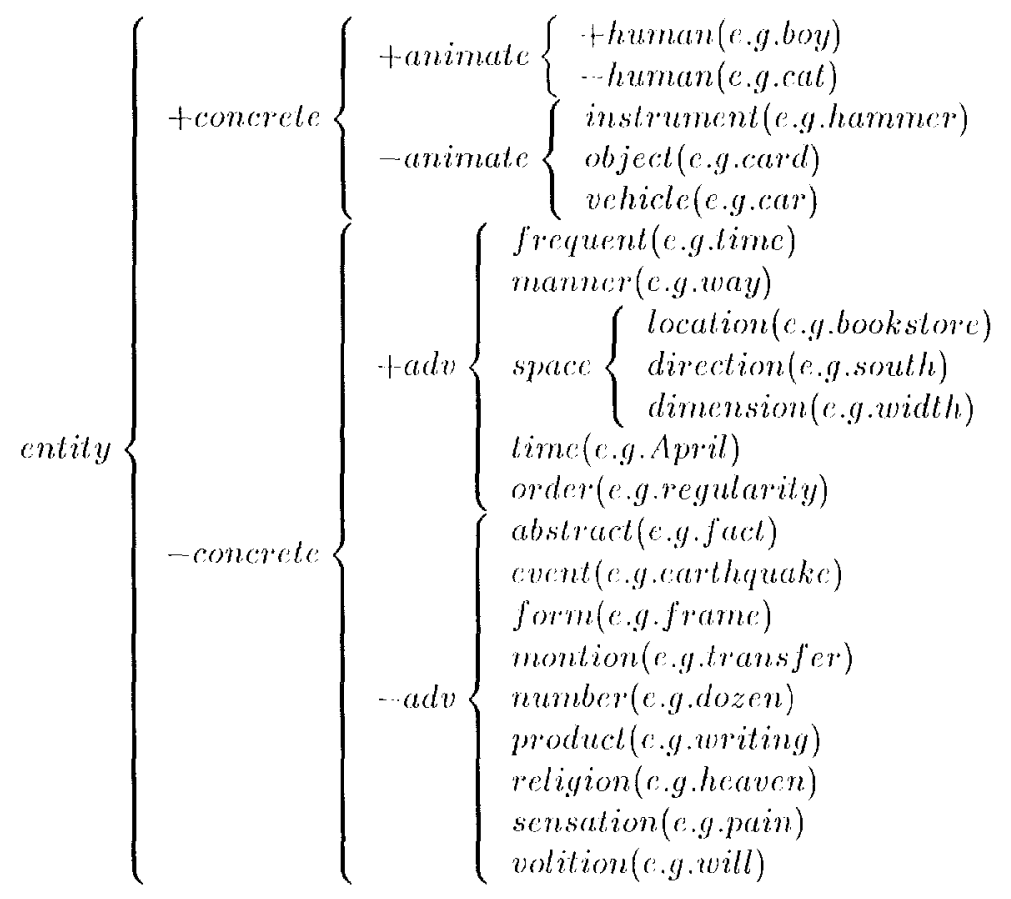

Figure 2: Somantics lags for Nouns. 
not misdetermined and this corresponds to the behavior to model refinement. However, many VPP's are not correctly resolved due to the rigidity of ruk-temples. Therefore, relaxing these rules will result in more correct VPP, but less correct NPP. Another difliculty comes from the assignment of semantic tags. As everyone knows the sense ambiguity is a serions problem, to assign unique semantic tag is hand. We plan to resolve this problem in the near future and to use the semantic-tanged corpus to tain the rule-templates.

\section{Concluding Remarks}

Prepositional phrases usually result in structural ambiguities and cost systems many resoures to resolve the attachnent. We develop a rule-based and M't-oriented model refinement algorithm to tackle this problem. We find PPP, SPP, VPP, and NPP are more realistic than only two attachment. choices in mathine translation. After large-scale experimentis, the results show that rule-based system is also useful for difficult problem like PP' at tachment. However, the determination of VP? is relatively difficult muder our algorithun. Another clifficulty is how to assign unique semantic tag to word. The resolution for these two problems will greatly improve the performance of this work.

\section{References}

V. Brili and P. Resnik. 1994. A Rule-Based Approach to Automated Prepositional Phrase Attachmont Disambignation. Procedings of COLING-94. page $1198-1204$.

K. H. Chen and II. H. Chen. 1992. Attachment and 'Transfer of Prepositional Phrases with Constraint Propagation. Compuler Proressing of Chiness: and Oriental Languages: An International fournal of the Chinese language Computer soctely, 6(2), page $123 \cdot 142$.

K. II. Chen and II. II. Chen 1994. Acquired Verb Subeategorization lirames. Procedings of the Second Conference for Natural Langauge. Processing (KONVENS-94), page 407410 . Vicolla, Austria.

k. H. Chen and II. II. Chen. 1995. Machine 'Translation: $\Lambda$ In Integrated Approach. Procedings of the sirth International Conference on Theortical and Methodological Issues in Machine Franslation, page 287294.

M. Ford, J. Brosnan and R. Kaplan. 1982. A Competence-Based Theory of Syntactic Clo sure. The Menlal Representation of Grammatiral Relations, J. Bresman, Eds., MIT' Press, page $727 \cdots 796$.
I. Lrazier. 1978. On Comprehending Sentenes: Syntactic Parsing Slrategies, I)octoral Dissortation, University of Connecticut.

D. Hindle and M. Rooth. 1993. Structural Ambiguity and Lexical Relations. Compulational linguistics, 19(1), page 103 120.

A. S. Hornby. 1989. Oxford Adeaned lacamer's Dictionary, Oxford Haiversity Press.

J. Kimball. 1973. Seven Prineiples of Suffare Structure Parsing in Nalural language. (oynation, 2, page. 15 17

C I. Riu, J. S. Chang and K. Y. Su loged 'Ther Somantic Seore Approach to the Disambigutation of PP'Atachment P'roblem. Procedings of hOCLING-90, page 2533270 . 'Laiwan, R.O.C'

M. Marcus, 13. Santorini, M. A. Marcinkiewic\% 1993. Buileling a Large Annotated Corpus of linglish: the Penn lroobank. (ompulatemal linguistics, 19(2), page 313330.

S. Shicber. 1983. Sentence Disambiguation by a Shifl-Redueed Parsing 'Pechnicpue. Procedtumgs

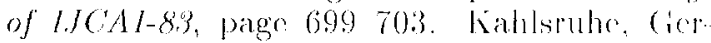
màny.

Y. Wilks, X. H. Huang and D). Jass. 1985. Synlax.

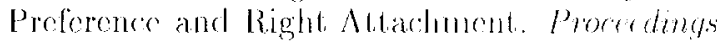
of l.J CA-85, page 779 -781. los Angeles, ('A

\section{Appendix}

The following lists rule-templates for PP. attachment. Hvery template (e) nsists of four ele ments $\langle V, N 1, P, N 2\rangle$. 'I'he curl brakelset pair do. notes OR, the underline denotes DON"I" ('ARE and $-\mathrm{r}$ denotes NO'I'.

I. Rule-template for SPP

1. 〈ـ, _, aboul, lime $\rangle$

2. 〈—, _, across, location $\rangle$

3. (—, after, lime)

4. 〈_, _, along, lorration $\rangle$

5. 〈—, _, among, location)

6. $\langle\ldots, \ldots$, at. $\{$ location, lime $\}\rangle$

7. 〈ـ, _, be fore, time $\rangle$

8. $\langle$ _, _, between, \{location, lime $\}\}$

9. $(\ldots$, _, by, time

10. 〈_, _., during, time $)$

11. $\{-, \ldots$, in, $\{$ location, thmo $\}\rangle$

12. 〈_, _., in front.of, location $\rangle$

13. __, near, location) 


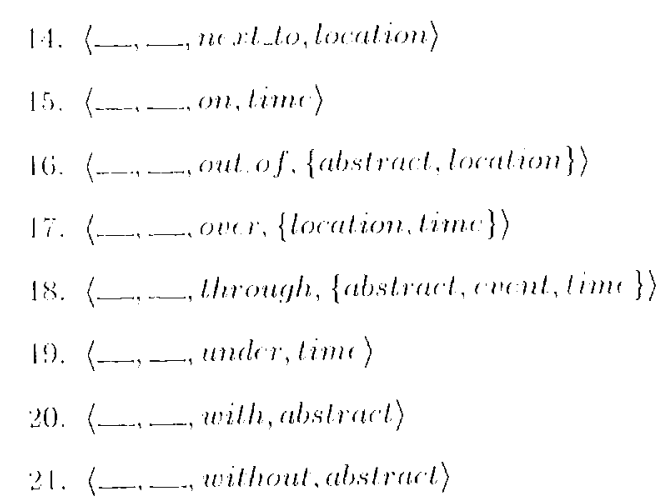

Il. Rulcentemplate for Vep

1. (motion,..., aboul, \{object,localion $\})$

2. (at_mant. _.., aboul, objorl)

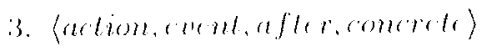

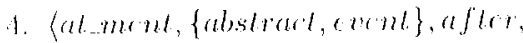
$\{$ anchl, no, $\lim \}\}$

5. $\langle$ moliom,__, arrosss, $\{$ loratiom, ots.jecl $\}\rangle$

(j. $\langle\{$ at nommern, ai nonmen $\}, \ldots . .$, along, \{location, objal $\}\}$

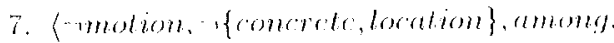
\{courrete, loration $\}$

\&. $\langle\{\{$ al nonmen, ai_nommen $\}, \ldots$, al. $\{$ animalr, objerelt\}\}

9. $\{\{$ abl nonmen, ai_nommon $\}, \ldots$, ab $\{$ torretion, objerl $\}\}$

10. (action, ancht, after, concerte)

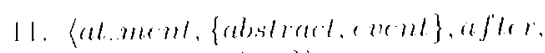

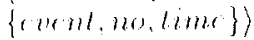

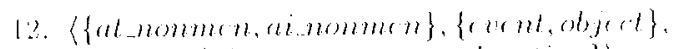

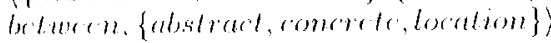

13. $\{$ at.nommen, ar nonmen\}, \{andal, objorl $\}$, beteecentime $\rangle$

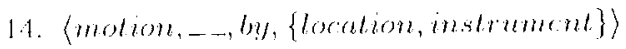

15. $\langle\ldots \ldots, b y, m a m m r\rangle$

16. 〈-motion, _., by, \{locration, object $\}\}$

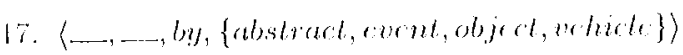

18. 〈_, _, by, antmale $\rangle$ passive voice

19. $\langle\ldots, \ldots$, for, lime $\rangle$

30. 〈molion,_, for, location $\rangle$

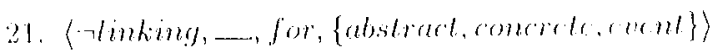

$22 .\langle\ldots, \ldots$, for, $\{$ ablatracl, edent, objert $\}\}$

23. $\left\{\_\ldots\right.$ - $\int o n$, rnimale $\left.\}\right\rangle$

24. $\langle\{$ molion, specech_tuct $\}, \ldots$, from, rnltity $\}$
25. $\{$ motion, _ in, $\{$ loration, instrument $\}\}$

$2(i .\langle\ldots, \ldots$, in. $\operatorname{tim}\rangle$

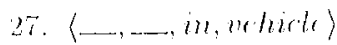

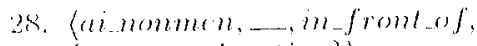
$\{$ comerte, loration $\}$

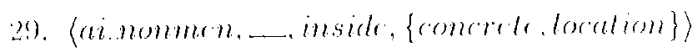

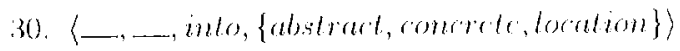

31. $\langle$ ard, - , like, - $\rangle$

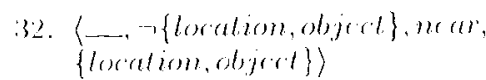

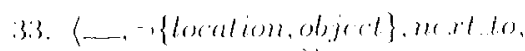
$\{$ location, abjert $\}\}$

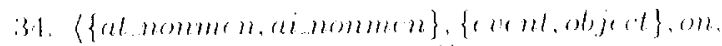

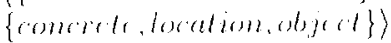

35. $\langle\ldots, \ldots, 0 n, \operatorname{coc}(n)\}$

36. 〈ـ, _., on_lo, \{lieration, objerel $\}\rangle$

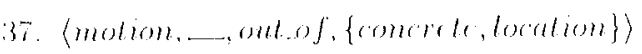

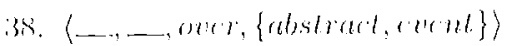

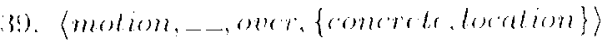

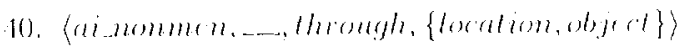

11. $\{\{a$ nommen, at_nomman\}, _. under, $\{$ ads stearl, ob.jorat $\}\rangle$

12. 〈_. - unlil, time

13. $\langle$ al nommon, _. with, instrumenl $\}$

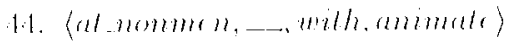

15. (at.nommen. _. Wilhoul. mstrumenl)

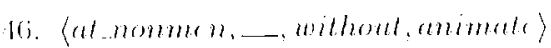

